\title{
CONSEQÜÊNCIAS DA SEGREGAÇÃO RESIDENCIAL PARA AS POLÍTICAS PÚBLICAS: o caso do atendimento básico em saúde em São Paulo ${ }^{1}$
}

\author{
Haroldo da Gama Torres* \\ Renata Bichir *
}

\section{INTRODUÇÃO}

Dialogando com algumas abordagens que procuram explicar as condições diferenciadas de acesso a políticas públicas, este artigo desenvolve o argumento de que variáveis de diversas naturezas - demográficas, institucionais, espaciais, relativas ao associativismo, entre outras - devem ser consideradas nesse tipo de análise, de modo a construir um cenário mais completo das situações de acesso a políticas públicas. Em especial, o artigo procura avaliar as conseqüências da segregação residencial, ou seja, da concentração espacial de certos grupos sociais, para as condições de acesso da população mais pobre do município de São Paulo à política de saúde, focalizando, especialmente, o atendimento básico de saúde.

* Doutor em Ciências Sociais pela Universidade de Campinas. CEBRAP - R. Morgado de Mateus, 615 (Vila Mariana) - São Paulo-SP - Brasil. Cep: 04015-051. hgtorres@uol.com.br

**Doutoranda em Ciência Política do IUPERJ. Pesquisadora do Centro de Estudos da Metrópole do CEBRAP renatambichir@yahoo.com.br

${ }^{1}$ Este trabalho foi apresentado preliminarmente no $5^{\circ}$ ENCONTRO DA ASSOCIAÇÃO BRASILEIRA DE CIÊNCIA POLÍTICA-ABCP, em Belo Horizonte, em julho de 2006, na ST06 - Políticas Públicas.
Para tanto, o artigo baseia-se em um survey realizado pelo Centro de Estudos da Metrópole (CEM-CEBRAP) em novembro de 2004, que serviu como instrumento para a avaliação dessa política. ${ }^{2} \mathrm{O}$ survey foi aplicado aos $40 \%$ mais pobres da população de São Paulo, de forma a distinguir situações de pobreza nas diferentes macro-regiões da cidade ${ }^{3}$ e, assim, considerar o fenômeno da segregação. Esse survey teve como objetivo verificar diversos aspectos das condições de vida da população pobre, destacando as condições de acesso a políticas públicas de educação, saúde, transferência de renda e infra-estrutura urbana. Os detalhes metodológicos sobre este survey são apresentados no Anexo I.

Assim, este artigo divide-se em cinco partes, incluindo a introdução. Na segunda seção, é apresentada uma discussão a respeito das diferentes abordagens explicativas para o acesso a políticas públicas. Com base nos dados fornecidos pelo survey, são apresentados, na terceira seção, os ní${ }^{2}$ Esse projeto teve o apoio do IPEA. Ver Figueiredo et al, 2005.

${ }^{3}$ O próprio recorte espacial do survey considerou a dimensão da segregação, uma vez que foram aplicados questionários em áreas de classe alta, média e baixa. Ver Anexo I. 
veis de cobertura observados no caso dos atendimentos básicos de saúde entre a população mais pobre. Considerando os elevados níveis de cobertura observados, foi construído um indicador mais exigente de acesso, apresentado na terceira seção. A quarta seção apresenta um modelo multivariado de análise - a árvore de CHAID -, com base nesse indicador sintético apresentado e em variáveis explicativas que procuraram cobrir as diferentes vertentes apresentadas na segunda seção. Por fim, são apresentadas as considerações finais, que destacam a complexidade envolvida no entendimento dos condicionantes do acesso da população mais pobre do município de São Paulo ao atendimento básico de saúde.

\section{PRINCIPAIS ABORDAGENS PARA O ACESSO A POLÍTICAS PÚBLICAS}

Antes de apresentar os dados relativos à cobertura do atendimento básico de saúde, é importante discutir, de forma breve, os principais elementos explicativos presentes no debate atual. A partir de um levantamento parcial da literatura brasileira sobre economia, sociologia, demografia e ciência política, visando a identificar os principais argumentos utilizados para explicar ou negar o acesso dos grupos de baixa renda às políticas públicas, identificamos quatro linhas interpretativas, que são aqui discriminadas para fins analíticos. Essa divisão não deve ser encarada de forma rígida, uma vez que alguns dos autores mencionados combinam, em sua interpretação, argumentos oriundos de mais de uma linha interpretativa.

Em uma primeira linha de interpretação, com maior influência na economia e na demografia, o acesso a serviços está associado às características dos indivíduos e das famílias, destacando-se, especialmente, suas características socioeconômicas. Essa abordagem considera que, além da renda, fatores como escolaridade dos indivíduos, escolaridade dos pais, idade e sexo seriam importantes elementos na explicação do nível de acesso a serviços públicos, uma vez que condicionariam, por exemplo, o grau de conhecimento individual a respeito da existência e das formas de acesso, o que afetaria o comportamento desses agentes, por meio de diversos mecanismos. Essas explicações estão presentes com freqüência, por exemplo, nos estudos que analisam as condições de acesso às políticas de educação e também em análises que visam a explicar os diferenciais no nível educacional das crianças de baixa renda (Barros et al. 2001; Menezes-Filho; Pazello, 2005).

Uma segunda linha de interpretação destaca as possíveis lógicas e interesses que o Estado (ou segmentos de sua burocracia) teria, ao fornecer ou não serviços às camadas mais pobres da população. Trata-se de uma abordagem que se desdobra em diferentes vertentes explicativas e alternativas. Em uma primeira vertente, de inspiração marxista, presente especialmente no debate brasileiro das décadas de 1970 e 1980, as áreas periféricas eram caracterizadas como locais desprovidos de investimentos públicos, marcados por precariedades extremas e pela ausência do Estado, relacionandose esse fenômeno à lógica de reprodução do capital (Kowarick, 1979).

Criticando essas explicações macroestruturais, começaram a surgir, na década de 1990, explicações que destacavam a presença de investimentos estatais nas áreas periféricas desde o final da década de 1970 e que buscavam entender os condicionantes dessa atuação estatal (Watson, 1992; Marques, 2000; Bueno, 2000). ${ }^{4}$ É parte desse conjunto de explicações, por exemplo, o modelo da relação entre investimentos públicos e ciclos eleitorais (Fizson, 1990; Ames, 1995), segundo o qual os investimentos públicos - especialmente aqueles destinados para as áreas mais pobres - seriam maiores nos períodos anteriores às eleições, de modo a gerarem retornos políticos (especialmente reeleição).

Outras explicações, relacionadas à lógica burocrática e à inércia do setor público, também integram esse conjunto de estudos, embora tenham

\footnotetext{
${ }^{4}$ Esses estudos contribuíram para destacar que as periferias não eram marcadas por um completo vazio institucional, mas ressaltavam que esses investimentos públicos não eram suficientes para reverter o padrão geral de privilégio de áreas centrais.
} 
sido mais utilizadas para elucidar por que os pobres passaram a ter acesso a determinadas políticas, e não para interpretar a heterogeneidade de acesso entre os pobres. Nesse contexto, insere-se, por exemplo, a tese da "seletividade hierárquica" das políticas, que destaca a influência da cultura técnica da burocracia de certos setores de políticas sobre a implementação das mesmas, fazendo com que elas sejam expandidas prioritariamente para as áreas mais ricas das cidades e, posteriormente, para as áreas mais periféricas (Marques, 2000).

Um terceiro eixo interpretativo diz respeito ao papel dos movimentos sociais e sua influência sobre a oferta de serviços públicos (Jacobi, 1989; Sader, 1988; Gohn, 1991). Ou seja, as políticas sociais seriam expandidas para as regiões mais pobres da cidade, após a pressão de grupos sociais organizados que, dessa forma, contribuiriam para alterar a direção dos investimentos estatais. Segundo Marques (2003), essas explicações configurariam um "modelo do conflito", mas poderiam ser também interpretadas dentro de uma abordagem que associa o acesso a serviços públicos ao papel de grupos de interesse.

Uma variação dessa terceira abordagem enfatiza a importância dos grupos de parentesco, o pertencimento a associações comunitárias e os vínculos com instituições religiosas ou laicas, como elementos que influenciam o acesso a serviços públicos, especialmente no âmbito de comunidades carentes (Gurza Lavalle; Castello, 2004; Almeida; D'Andrea, 2004). Esses autores destacam ainda o papel desempenhado pelas práticas associativas - especialmente religiosas - na atenuação dos efeitos da exclusão, especialmente no caso da inserção no mercado de trabalho. Essa literatura faz parte do universo interpretativo das teorias sobre capital social, embora o tema possa se desdobrar em diferentes recortes analíticos (Putnam, 2000). Essa dimensão da importância das redes de relações está presente na literatura desde a descrição dos processos de produção dos loteamentos periféricos das décadas de 60 e 70 (Santos, 1985).

Finalmente, a quarta vertente explicativa destaca o papel da segregação residencial, ou seja, da concentração espacial de certos grupos sociais, no acesso a serviços públicos. Para esses autores (tais como Villaça, 2000; Marques; Torres, 2005), não se trata apenas de reafirmar a existência de diferenciais socioeconômicos no espaço, mas de defender a existência de uma dimensão espacial que interfere no funcionamento das políticas públicas. A residência em locais altamente segregados teria como principais conseqüências o isolamento em relação às redes sociais e econômicas mais relevantes e a exposição a diversas condições de risco, que gera uma série de "externalidades negativas" com efeitos significativos sobre os circuitos de reprodução da pobreza (Torres, 2004). Essas análises indicam, por exemplo, que indivíduos de condições sociais idênticas, mas localizados em regiões distintas da cidade, tendem a ter acessos bastante diferenciados a serviços, bem como ao mercado de trabalho (Torres, et al. 2005; Durlauf, 2001; Brooks-Gunn; Duncan, 1997).

Cabe ainda mencionar uma abordagem, pouco presente na literatura brasileira, que não pode ser facilmente identificada com nenhuma dessas matrizes interpretativas, pois busca integrar várias dessas perspectivas. Essa linha de interpretação destaca o papel desempenhado pelas chamadas "estruturas de oportunidades", configuradas em cada comunidade (proporcionadas pelo mercado, pelo Estado e pela própria comunidade), que podem facilitar ou constranger o acesso a políticas públicas, além de contribuírem para processos de reversão ou reprodução das condições de pobreza (Kaztman; Filgueira, 1999). Também faz parte dessa vertente explicativa uma noção desenvolvida inicialmente por Moser (1998), que destaca os ativos possuídos pelas populações mais pobres - como sua malha de relações sociais, os tipos de trabalhos que desenvolvem no âmbito doméstico, suas posses materiais, etc. - que devem ser bem “gerenciados", para que não se tornem vulneráveis. Como apresentado acima, fica claro que a interpretação dos diferentes graus de acesso dos mais pobres a políticas públicas não é trivial, sendo, ao contrário, objeto de um complexo debate. Os dados obtidos por meio do survey permitem 
testar alguns desses argumentos no caso do acesso do acesso à política de saúde, como será apresentado. A seguir são apresentados os níveis de cobertura do atendimento básico de saúde.

\section{NÍVEIS DE COBERTURA DO ATENDIMENTO BÁSICO DESAÚDE}

Optamos por avaliar, no survey, as condições de atendimento básico de saúde que estão sendo oferecidas à população, ou seja, os atendimentos de rotina que são oferecidos nos postos de saúde e mesmo em hospitais, por meio de consultas básicas. Sendo assim, foram excluídos procedimentos mais complexos ou de emergência, que sabidamente tendem a ter uma distribuição espacial mais concentrada. Nesse caso, a população total analisada refere-se aos domicílios pobres que tiveram algum membro procurando atendimento de saúde no último ano, em posto de saúde ou em hospital que não pronto socorro. Foram avaliados diversos aspectos, tais como os tipos de locais que foram procurados para atendimento de saúde, o tempo de espera para obtenção de atendimento, a distância percorrida para obtenção desse atendimento, as dificuldades observadas e a necessidade de ajuda de terceiros, além da avaliação dos serviços.

Em primeiro lugar, os dados do survey indicam que, em 91,0\% dos domicílios mais pobres do município, algum dos membros da família teve de procurar algum tipo de atendimento de saúde de rotina no último ano, número bastante expressivo, sendo que, em 49,5\% dos casos, o atendimento tinha ocorrido há até um mês antes da pesquisa (novembro de 2004). Também nesse caso, observa-se um elevado nível de cobertura do sistema público municipal de saúde. Dentre aqueles que declararam ter membros da família buscando atendimento de rotina no último ano, 57,2\% procuraram postos de saúde e 16,0\% hospitais. Isso também indica o sucesso da hierarquização do sistema, que prevê atendimentos básicos em postos de saúde, e também o fato de que as famílias mais pobres recorrem de forma significativa aos serviços públicos.
Analisando essa informação por tipo de região, verifica-se que as pessoas residentes em áreas periféricas recorrem bem mais aos postos de saúde públicos e menos a hospitais públicos: $60,2 \%$ das pessoas nas áreas periféricas recorrem a postos de saúde, contra 47,2\% nas áreas centrais. Esses dados são consistentes com a própria distribuição espacial dos equipamentos de saúde, uma vez que os hospitais tendem a estar concentrados nas áreas centrais do município. ${ }^{5}$

Após a seleção daqueles que recorreram a postos de saúde ou a hospitais públicos para atendimento de rotina, foram verificados outros aspectos que permitem caracterizar as condições de atendimento oferecidas. Em primeiro lugar, avaliou-se o tempo de espera para o atendimento, diferenciando-se as situações em que houve marcação de consulta daquelas em que não houve, sendo que, em 71,6\% dos casos, houve agendamento da consulta. Nas situações de atendimento com consulta marcada, o tempo médio de espera foi de 39 dias, e a mediana foi de 30 dias, sendo que a variação é bastante grande, de um dia a um ano. Esse tempo médio de espera é significativamente mais elevado para aqueles que moram em áreas periféricas e em áreas de favela, indicando a relevância dessas variáveis territoriais. De acordo com o esperado, o tempo de espera é significativamente maior no caso de consulta marcada em hospital (56 dias em média) do que no caso de consultas marcadas em postos de saúde (média de 36 dias).

Nas situações sem marcação de consulta, o tempo médio de espera foi de aproximadamente 1 hora, atingindo um máximo de 12 horas. Nesse caso, é interessante notar que não há diferenciais por região, conforme as tendências usualmente esperadas - uma vez que, nas áreas centrais, as pessoas esperam mais do que nas áreas periféricas. Porém o fato de o domicílio estar localizado em área de favela ${ }^{6}$ aumenta o tempo de espera para

\footnotetext{
É importante observar que uma completa avaliação das condições de acesso aos serviços de saúde pública de rotina deveria envolver a análise das condições da oferta desses serviços, o que não pôde ser captado por meio dos dados do survey e foge ao escopo deste artigo.

${ }^{6}$ Por meio de ferramentas de geoprocessamento, todos os questionários do survey foram endereçados por meio do
} 
a consulta sem agendamento, que passa para $1 \mathrm{~h} 20 \mathrm{~min}$. Novamente, de acordo com o esperado, o tempo de espera para atendimento em hospital é muito maior do que o tempo de espera para atendimento em posto de saúde: 2 horas em média nos hospitais, contra 40' minutos em média nos postos de saúde.

Considerando a distância percorrida para obtenção de atendimento, verifica-se que as pessoas tiveram de se deslocar, em média, 738 metros, deslocamento pequeno, que está relacionado à informação de que a maioria das pessoas recorreu ao posto de saúde mais próximo de suas casas. O deslocamento máximo observado foi de $6 \mathrm{~km}$. De acordo com o esperado, o deslocamento médio é maior no caso daqueles que foram a hospitais públicos, ao invés de postos de saúde: quem busca atendimento em hospital tende a percorrer em média $1 \mathrm{~km}$, enquanto aqueles que buscam atendimento em posto de saúde percorrem em média 700m. ${ }^{7}$

Atentando para as maiores dificuldades observadas na obtenção de atendimento de saúde, destaca-se, em primeiro lugar, a dificuldade ou a demora na marcação das consultas, seguida pela demora no atendimento. A Tabela 1 apresenta essas informações. Esses resultados reforçam a relevância do indicador sintético de acesso adotado, como será demonstrado.

Considerando a distinção entre os locais de atendimento, observa-se que, no caso dos atendimentos realizados em postos de saúde, a maior dificuldade percebida é a demora para a marcação de consulta. No caso daqueles que recorreram a hospitais, a demora na marcação de consultas também é o maior problema percebido, porém destaca-se, em segundo lugar, a distância do local de

CEP. Desse modo, foi possível associar os dados obtidos com informações oriundas de outras fontes, nesse caso, com dados provenientes da base de favelas desenvolvida pelo CEM (Marques; Torres; Saraiva, 2003).

${ }^{7}$ Devido a dificuldades enfrentadas pela população na estimação de distância através dos parâmetros comumente utilizados (metros ou quilômetros), optamos por estimar as informações relativas à distância por meio de quarteirões. De modo a fazer as devidas conversões, considerou-se que um quarteirão eqüivale a aproximadamente 100 metros e que $1 \mathrm{~km}$ é equivalente a aproximadamente dez quarteirões. Esse procedimento mostrou-se de grande utilidade na aplicação do survey.
Tabela 1 - Maior dificuldade no atendimento de saúde. Município de São Paulo, 2004

\begin{tabular}{lc}
\hline Maior dificuldade & \% Válido \\
\hline O local de atendimento é distante/acesso difícil & 11,5 \\
\hline É difícil ou demora para marcar a consulta & 33,9 \\
\hline Filas grandes & 7,3 \\
\hline Burocracia & 4,2 \\
\hline Horário ruim & 0,8 \\
\hline O tempo da consulta é pequeno & 1,0 \\
\hline Local mal equipado & 1,4 \\
\hline Funcionários com má vontade & 2,7 \\
\hline Remédios caros & 2,7 \\
\hline Demora no atendimento & 13,4 \\
\hline Falta de médicos & 8,9 \\
\hline Falta de médicos especialistas & 3,7 \\
\hline Médicos com má vontade & 4,5 \\
\hline Outra & 4,0 \\
\hline Total & 100,0 \\
\hline
\end{tabular}

Fonte: CEM-Cebrap/Ibope. Survey de acesso da população mais pobre de São Paulo a serviços públicos. Nov. 2004.

atendimento, o que é condizente com a maior concentração espacial dos hospitais. Nas áreas de classe baixa e também nas áreas de favela, os principais problemas apontados são as dificuldades na marcação de consulta e a falta de médicos.

Analisando os mecanismos utilizados pela população para a obtenção de atendimento, verificase que a grande maioria das pessoas $(83,2 \%)$ não precisou de ajuda de ninguém no momento de buscar atendimento de saúde, um dado bastante positivo, pois revela que conseguem acessar o sistema sem ter de passar por intermediários. E, quando tiveram de recorrer à ajuda de alguém, foi a algum agente de saúde, o que indica que, mesmo quando ocorre ajuda, ela é "institucionalizada". Analisando essa informação por tipo de região, nota-se que aqueles que residem em áreas periféricas tenderam a recorrer mais à ajuda de alguém do que os habitantes da área central: $19 \%$ contra $9,4 \%$, sendo que os residentes em áreas de classe baixa recorrem mais à ajuda de parentes e vizinhos do que os habitantes das áreas das classes média e alta. Não há diferenças significativas de acordo com o local de atendimento.

Assim, de maneira geral é possível dizer que o acesso ao atendimento básico de saúde apresen- 
ta cobertura razoável, mesmo entre a população mais pobre do município, e também se encontra institucionalizado. Esse cenário nos levou à construção de um indicador mais exigente de acesso, como explicado a seguir.

\section{Indicador sintético}

O indicador sintético foi construído, visando a qualificar as condições de acesso à política de saúde pública. Na construção desse indicador, optou-se por avaliar a "espera para obtenção de uma consulta", uma vez que essa foi a principal dificuldade apontada pelos entrevistados (ver Tabela 1). Assim, foram diferenciadas as situações em que houve agendamento prévio (maioria das situações) e aquelas em que o atendimento não foi agendado (foi realizado no mesmo dia), de modo a compor um indicador final que sintetizasse essas duas informações.

Quando a consulta foi agendada previamente, os valores do indicador são: 1 para mais de 30 dias, 0,5 para 16 a 30 dias e 0 para até 15 dias, ou seja, quanto menor o valor do índice, menor o tempo de espera. No caso de não ter ocorrido o agendamento prévio, considerou-se o valor 1 para mais de uma hora de espera para ser atendido e 0 para menos de uma hora. Todos esses cortes foram estabelecidos a partir da análise das distribuições estatísticas dessas variáveis. A variável final utilizada no modelo multivariado representa a média para o tempo de espera para atendimento de saúde de rotina, sendo que quanto maiores os valores desse indicador, maior o tempo de espera

Tabela 2 - Indicador de tempo de espera para atendimento básico de saúde, segundo tipo de regiảo.

Município de São Paulo, 2004

\begin{tabular}{lcc}
\hline Região & Média & Desvio padrão \\
\hline Periférica & 0,50 & 0,42 \\
\hline Intermediária & 0,46 & 0,43 \\
\hline Central & 0,42 & 0,43 \\
\hline Total & 0,48 & 0,42 \\
\hline \multicolumn{3}{l}{ Fonte: CEM-Cebrap/lbope. Survey de acesso da populaçáo mais pobre de } \\
Sáo Paulo a serviços públicos. Nov. 2004.
\end{tabular}

para o atendimento (considerando-se consultas agendadas e não agendadas de forma agregada).

A Tabela 2 apresenta a distribuição desse indicador sintético de tempo de espera por tipo de região. Nota-se que, nas áreas periféricas, o tempo de espera para a consulta de saúde tende a ser maior que no caso das áreas centrais.

Também possível observar, de acordo com a Tabela 3, que a espera é maior no caso de atendimentos básicos de saúde ocorridos em hospital, seguindo o sentido geral dos indicadores desagregados por consulta "agendada" e "não agendada", apresentados anteriormente.

Contudo verifica-se que a variabilidade dos escores de tempo médio de espera é baixa, o que novamente aponta para a quase universalização do acesso ao atendimento básico de saúde, mesmo entre a população mais pobre do município. Esses resultados são aprofundados no modelo apresentado a seguir.

\section{O modelo de análise}

Nesta seção, apresentamos o modelo de análise elaborado para associar diferentes variáveis explicativas para o acesso das camadas mais pobres do município de São Paulo à saúde. Esse modelo é baseado na chamada "árvore de CHAID" e é bastante útil em análises exploratórias, quando as associações entre as variáveis de interesse não são bem conhecidas, permitindo detectar interações de modo mais fácil do que no caso de uma regressão. Uma interessante aplicação do método é a análise dos principais condicionantes

Tabela 3 - Indicador de tempo de espera para
atendimento básico de saúde, segundo local de
atendimento.
Município de São Paulo, 2004
\begin{tabular}{lcc}
\hline Local de atendimento & Média & Desvio padrão \\
\hline Hospital & 0,51 & 0,46 \\
\hline Posto de saúde & 0,47 & 0,41 \\
\hline Total & 0,48 & 0,42 \\
\hline Fonte: CEM-Cebrap/lbope. Survey de acesso da população mais pobre de \\
São Paulo a serviços públicos. Nov. 2004.
\end{tabular}


do acesso a políticas públicas, conforme utilizado hipóteses relacionadas à segregação residencial, à em Figueiredo et al. (2005). Para maiores informa- participação social, ao tipo de equipamento públições sobre essa metodologia, consultar o Anexo II. co, ao comportamento político e às características

A Tabela 4, abaixo, apresenta as variáveis individuais. ${ }^{8}$ No caso de variáveis institucionais, explicativas testadas no modelo.

Embora não seja possível resumir nessas referentes à natureza dos serviços oferecidos, foi variáveis todos os fatores explicativos mencionatestado o impacto da diferenciação entre atendidos na segunda seção deste artigo, foi considerado mento em postos de saúde ou em hospitais públium amplo espectro de temas consistentes com as cos. Variáveis relativas à preferência partidária e ao associativismo foram consideradas como próxis

Tabela 4 - Variáveis considerados no modelo.

Município de São Paulo, 2004

\begin{tabular}{|c|c|}
\hline Variáveis de vizinhança ${ }^{1}$ & Atributos do responsável/familiares \\
\hline Macro-Região de residência & Faixa etária do responsável/cônjuge \\
\hline - Área Central (com renda média alta) & -18 a 29 Anos \\
\hline - Área Intermediária (com renda média intermediária) & -30 a 49 Anos \\
\hline - Área Periférica (com renda média baixa) & - 50 Anos e Mais \\
\hline Renda média do responsável do domicílio (raio de $3 \mathrm{~km})^{2}$ & Sexo do responsável/cônjuge \\
\hline - Até 5 Salários Mínimos & - Masculino \\
\hline - Mais de 5 a 10 Salários Mínimos & - Feminino \\
\hline - Mais de 10 Salários Mínimos & Escolaridade do Responsável/Cônjuge \\
\hline - Sem informação & - Fundamental incompleto \\
\hline Domicílios localizados em favelas ou áreas próximas $(100 \mathrm{~m})^{3}$ & - Fundamental completo \\
\hline$-\operatorname{Sim}$ & - Médio completo ou superior \\
\hline - Não & Renda familiar per capita (sal. mínimo 11/2004) \\
\hline Indicadores de Participação Social/Religiosa & - Até 0,5 Salário Mínimo \\
\hline $\begin{array}{l}\text { Freqüência a atividades associativas religiosas } \\
\text { atividades associativas de cunho religioso }\end{array}$ & - Mais de 0,5 a 1 Salário Mínimo \\
\hline - Pelo menos quinzenalmente & - Mais de 1 a 2 Salários Mínimos \\
\hline - Mais que quinzenalmente ou não freqüenta & - Mais de 2 a 5 Salários Mínimos \\
\hline Freqüência a associações não religiosas & - Mais de 5 Salários Mínimos \\
\hline - Pelo menos anualmente & Cor do responsável \\
\hline - Mais que anualmente, ou não freqüenta & - branco \\
\hline Variáveis Políticas & - preto e pardo \\
\hline Influência dos acontecimentos politicos sobre a vida & Tipo de serviço público \\
\hline - Acha que influenciam sua vida & Tïpo de Escola Pública \\
\hline - Acha que não influenciam sua vida & - Municipal \\
\hline - Não sabe/Não informou & - Estadual \\
\hline Preferência por partido político ${ }^{4}$ & Tipo de equipamento de saúde \\
\hline $\begin{array}{l}\text { - Com preferência partidária } \\
\text { Preferência pelo PT } \\
\end{array}$ & - Hospital \\
\hline - Sem preferência partidária & - Posto de Saúde \\
\hline \multicolumn{2}{|c|}{$\begin{array}{l}\text { Fonte: CEM-Cebrap/lbope. Survey de acesso da população mais pobre de } \\
\text { São Paulo a serviços públicos. Nov. 2004. } \\
{ }^{1} \text { Essas variáveis foram constrúdas, com a utilização de ferramentas de geoprocessamento (SIG), uma vez que os dados coletados no survey } \\
\text { foram endereçados por meio do CEP dos domicíios. } \\
{ }^{2} \text { Essa variável, também denominada de "ambiente local", procurou testar o impacto da micro-segregação sobre o acesso a políticas públicas, } \\
\text { avaliando o peso das características do entorno de cada área de ponderação - especialmente renda e escolaridade. A varíavel foi construída } \\
\text { por meio de ferramentas de geoprocessamento, com base nos setores censitários de } 2000 \text {. } \\
{ }^{3} \text { Essa variável também fó construída por meio de ferramentas de geoprocessamento, através do cruzamento da base de favelas desenvolvidas } \\
\text { pelo CEM (ver Marques; Torres; Saraiva, 2003) com os dados do survey. Devido a problemas no endereçamento dos questionários do survey } \\
\text { (que foi realizado somente com base no CEP dos domicílios entrevistados), optou-se por considerar os domicílios localizados dentro de um raio } \\
\text { de } 100 \mathrm{~m} \text { das áreas de favela. } \\
{ }^{4} \text { O percentual de pessoas que declarou preferência por algum partido foi de } 59,0 \% \text {, dos quais } 27,1 \% \text { declararam simpatia ao PT e e 8,0\% } \\
\text { declararam simpatia ao PSDB. Os demais, devido à baixa declaração de preferência, foram agregados. }\end{array}$} \\
\hline
\end{tabular}

${ }^{8}$ Os questionários foram aplicados somente aos responsáveis pelo domicílio, do sexo masculino ou feminino, que forneceram informações sobre outros membros da famíforneceram informações sobre outros membros da famítadas referem-se às características dos chefes de domicílio. 
do grau de acesso a informações e pertencimento a diversos tipos de redes disponíveis para a população de mais baixa renda do município.

Como variável dependente do modelo, foi utilizado o indicador sintético de tempo de espera para atendimento básico de saúde, já apresentado. O modelo de acesso elaborado, no caso desse indicador, é apresentado na Figura 1, a seguir.

Quando consideramos os resultados do modelo, podemos observar, em primeiro lugar, a relevância da região de residência: o grupo de fa- mílias que reside na região central do município espera menos tempo para ser atendido. Ou seja, a dimensão territorial é aquela que tem maior relevância na diferenciação das condições de acesso a essa política, resultado condizente com várias pesquisas realizadas no âmbito do Centro de Estudos da Metrópole (Marques; Torres, 2005; Figueiredo et al, 2005).

Dentre o grupo de moradores da área central, aqueles que recorreram ao atendimento em postos de saúde foram atendidos em menos tem-

\section{Figura 1 - Modelo CHAID para o indicador presteza no atendimento básico de saúde}

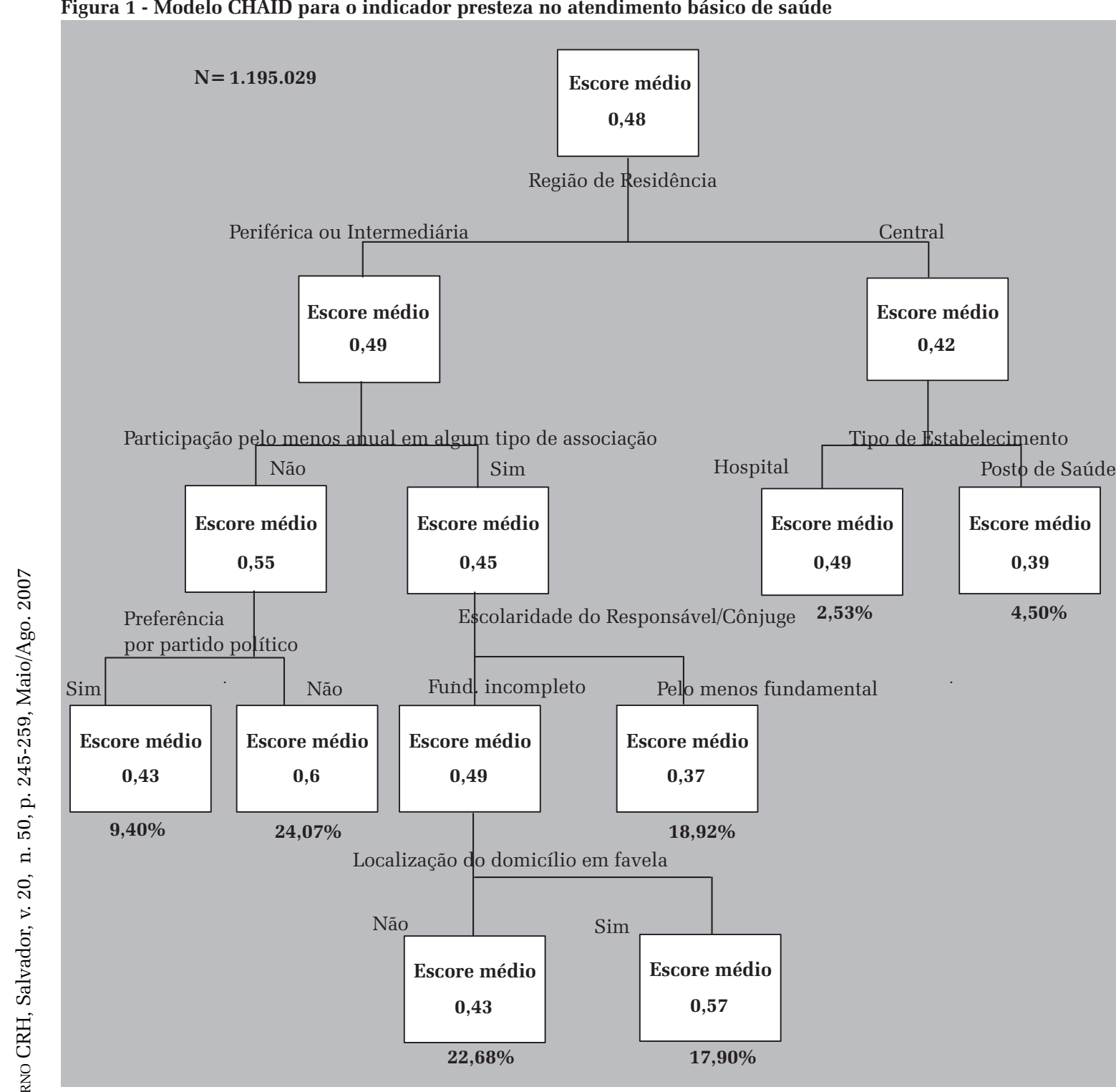

Fonte: CEM-Cebrap. Survey de acesso da população mais pobre de São Paulo a serviços públicos. Nov. 2004. Nota: Nível de Significância de 5\%. 
po, o que provavelmente pode ser explicado pela menor demanda por atendimento nessa região, especialmente se considerarmos que a concentração de pobres (e de habitantes em geral) é menor nessas áreas, fazendo com que a pressão sobre o sistema de saúde seja menor, e as "respostas" sejam mais rápidas. Esse resultado pode indicar também que os médicos permanecem menos tempo nos postos de saúde das áreas periféricas, conforme queixa dos próprios entrevistados. Esse tempo de permanência mais reduzido tem o mesmo efeito de aumento da pressão de demanda sobre os serviços nessas áreas.

Nas áreas periféricas e intermediárias, onde o tempo de espera para atendimento de saúde é maior, observa-se que a participação pelo menos anual em algum tipo de associação civil que não religiosa é uma variável relevante: famílias cujos membros participam de associações esperam menos para serem atendidas, provavelmente devido a efeitos de rede (maior acesso a informações sobre o funcionamento do sistema, maior contato com pessoas que podem facilitar o acesso, etc.). Entre as famílias que participam de alguma associação, o tempo de espera é menor para aqueles que têm maior escolaridade (pelo menos ensino fundamental completo) - o que novamente pode estar indi- cando maior acesso a informações sobre o funcionamento do sistema de saúde e sobre mecanismos de acesso - do que para aqueles que têm menos escolaridade. Entre os que têm menor escolaridade, aqueles que residem em favelas têm condições de acesso ainda piores (esperam mais para serem atendidos). Entre aqueles que não participam de nenhuma associação não religiosa, o acesso é melhor para as famílias que declararam ter preferência por algum partido político, novamente podendo indicar efeitos de rede sobre as condições diferenciais de acesso. Esses resultados são sistematizados na Tabela 5.

Resumindo, pode-se dizer que os condicionantes do acesso ao atendimento básico de saúde são bastante diversos, sendo destacada a relevância da dimensão territorial, além da dimensão institucional - no caso, a diferenciação entre atendimentos que ocorrem em hospitais e atendimentos em postos de saúde. As melhores condições de acesso são observadas no caso de famílias que residem na área central e que recorrem a postos de saúde, e também entre as famílias que residem em áreas periféricas ou intermediárias e participam de associações, além de seus membros possuírem ensino fundamental completo - esses grupos são os que obtêm atendimento de saúde de forma mais

Tabela 5 - Resumo do modelo de acesso para o indicador de tempo de espera para atendimento. Município de São Paulo, 2004

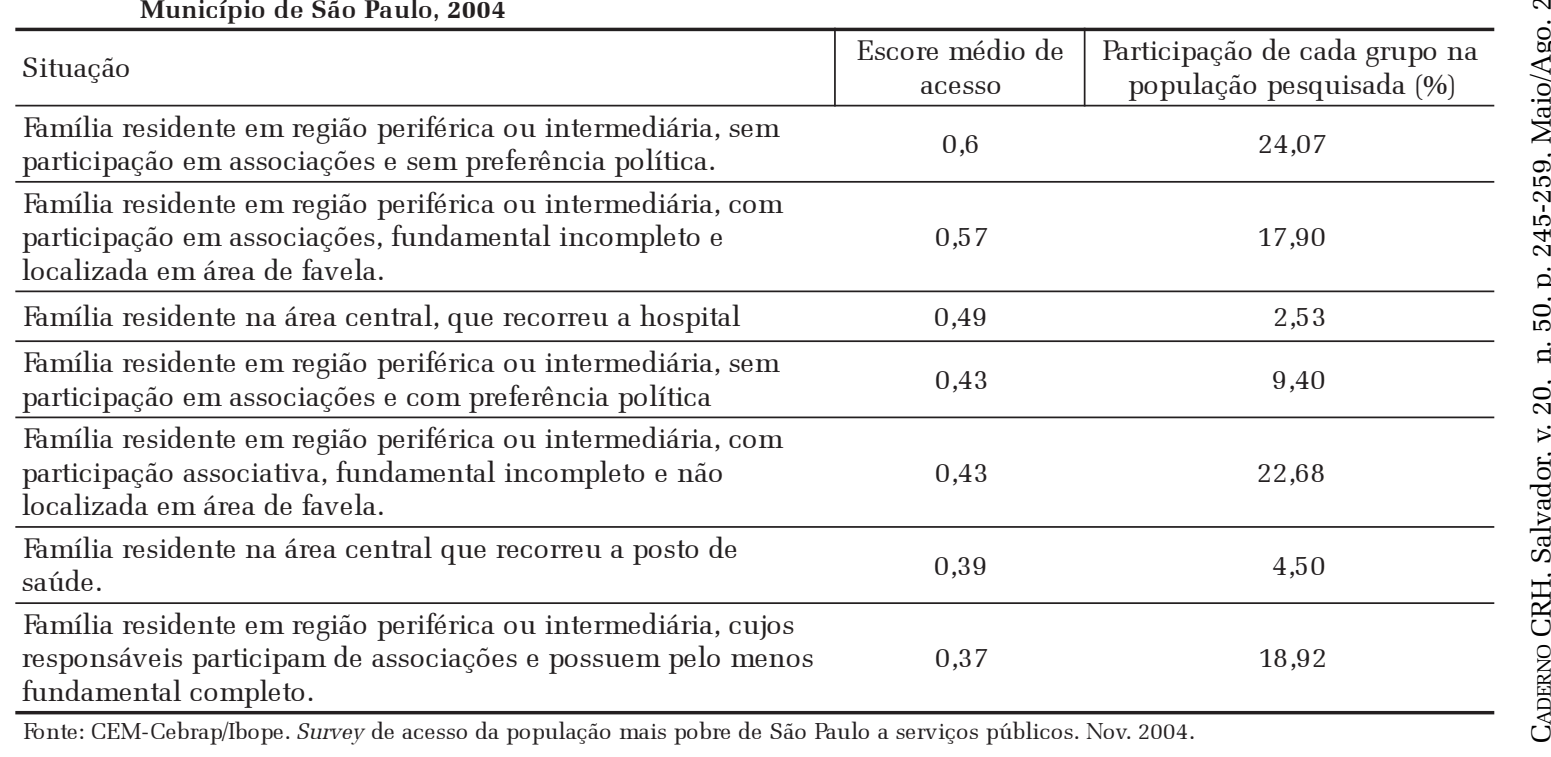


rápida. No extremo oposto, com as piores condições de acesso, estão as famílias que residem em áreas periféricas e cujos membros não declaram preferência partidária e nem participam de associações.

\section{CONSIDERAÇÕES FINAIS}

Em primeiro lugar, destacam-se os elevados níveis de coberturas do sistema básico de saúde pública, mesmo entre a população mais pobre do município de São Paulo e também a institucionalização dos mecanismos de acesso. Esse cenário aponta para a necessidade de construção de indicadores mais exigentes, que consigam captar dimensões qualitativas de acesso para além da cobertura das políticas.

Entretanto, quando analisamos o modelo multivariado elaborado com base no indicador sintético de tempo de espera para obtenção de consulta básica de saúde, verificamos a grande complexidade das condições de acesso, uma vez que variáveis mobilizadas por distintas matrizes teóricas mostraram-se relevantes na explicação dos diferentes níveis de acesso.

Foi demonstrado que as variáveis territoriais induzem as maiores variações no acesso a serviços básicos de saúde, sendo que áreas centrais tendem a ter melhores condições de acesso. Por um lado, esse resultado relaciona-se com as diferentes dinâmicas demográficas que ocorrem nesses espaços: nas áreas centrais, há menor demanda pelos serviços básicos de saúde, uma vez que há decréscimo populacional nessas áreas, e a população tem maior idade média; nas áreas periféricas, a pressão sobre o sistema é muito maior. Além disso, por outro lado, é possível observar certa concentração de equipamentos de saúde mais especializados nas áreas mais centrais do município (CEM-CEBRAP/SAS-PMSP, 2004), o que traz impactos significativos para as condições de acesso dos moradores de áreas periféricas.

Ainda no caso das variáveis territoriais, destaca-se o impacto negativo da localização dos domicílios em áreas de favela, sobretudo porque parecem refletir efeitos da pressão demográfica sobre os equipamentos de saúde, derivados da dinâmica populacional local e do tempo de permanência dos médicos na unidade de serviço. Tal efeito é particularmente importante em áreas de maior pressão da demanda, como as áreas periféricas.

Em segundo lugar, no caso das áreas centrais, o modelo destacou uma dimensão institucional, qual seja o tipo de unidade de serviço acessado, posto de saúde ou hospital, sendo que o tempo de espera tende a ser menor no caso dos atendimentos ocorridos em postos de saúde. Esse resultado é condizente com a hierarquização do sistema público de saúde.

Foram observados ainda impactos significativos, derivados tanto de variáveis de participação associativa, quanto no caso de preferência política. O papel tanto da participação associativa, quanto da preferência partidária no acesso a serviços pode ser interpretado como efeitos de redes de relações sociais que facilitam o acesso a informações sobre o programa em questão e sobre mecanismos de obtenção dos serviços.

Variáveis individuais, relativas aos atributos das famílias, não se mostraram significantes na diferenciação das condições de acesso a serviços básicos de saúde, à exceção da variável de escolaridade, que apresentou impacto limitado. Isso não significa que essas variáveis não tenham relevância no caso de outras políticas. Além disso, devido ao próprio recorte do survey - aplicado somente àqueles com renda familiar inferior a $\mathrm{R} \$ 1.100,00$, controlando-se sexo e idade dos responsáveis segundo cotas - o efeito das variáveis individuais sobre os diferenciais de acesso foi minimizado.

Todos esses resultados apontam para a complexidade dos fenômenos envolvidos na caracterização dos diferenciais de acesso da população mais pobre do município e para a necessidade de um olhar cuidadoso para as dinâmicas envolvidas no acesso à política de saúde.

(Recebido para publicação em setembro de 2006) (Aceito em agosto de 2007) 


\section{REFERÊNCIAS}

ALMEIDA, R.; D'ANDREA, T. Pobreza e redes sociais em uma favela paulistana. Novos Estudos CEBRAP, São Paulo, n. 28, mar. 2004.

AMES, B. Electoral strategy under open-list proportional representation. American Journal of Political Science, Washington, v. 39, n. 2, 1995.

BARROS, Ricardo Paes et al. Determinantes do desempenho educacional no Brasil. Textos para Discussão n. 834 Rio de Janeiro, IPEA, 2001.

BUENO, L. Projeto e favela: metodologia para projetos de urbanização. São Paulo: 2000 Tese (Doutorado) - Fau/ USP.

BROOKS-GUNN, J.; DUNCAN, G. (Eds.) Neighborhood poverty policy. Implications in studying Neighborhoods. New York: Russell Sage Foundation, 1997. v. 2.

CEM-CEBRAP/ SAS-PMSP. Mapa da vulnerabilidade social da população da cidade de São Paulo. São Paulo: SESC-SP, 2004.

DAHL, R. Who governs? Democracy and power in an American city. New Haven: Yale University Press, 1961.

DURLAUF, S.N. The membership theory of poverty: the role of group affiliations in determining socioeconomic outcomes. In: DANZIGER, S. H.; HAVERMAN, R.H. Understanding poverty. New York: Russell Sage., 2001. p. 392-416.

FARIA, V. A Conjuntura social brasileira: dilemas e perspectivas. Novos Estudos CEBRAP, São Paulo, n.33, 1992.

FIZSON, J. A política nacional de saneamento de 1968 a 1984: o caso do Planasa. 1990. Rio de Janeiro: Dissertação (Mestrado) - ENSP/FIOCRUZ.

FIGUEIREDO, A. et al. Relatório final do Projeto BRA/04/ 052 - Rede de pesquisa e desenvolvimento de políticas públicas: REDE-IPEA II. Rio de Janeiro: 2005. Mimeo.

GOHN, M. Movimentos sociais e luta pela moradia. São Paulo: Loyola, 1991.

GURZA LAVALLE, A.; CASTELLO, G. As benesses deste mundo associativismo religioso e inclusão socioeconômica. Novos Estudos CEBRAP, n. 28, mar. 2004.

JACOBI, P. Movimentos sociais e políticas públicas. São Paulo: Cortez , 1989.

KAZTMAN, R.; FILGUEIRA, C. Marco conceptual sobre activos, vulnerabilidad y estrutura de oportunidades. Montevideo: Cepal, 1999.
KOWARICK, L. A espoliação urbana. Rio de Janeiro: Paz e Terra, 1979.

MARQUES, E. Estado e redes sociais - permeabilidade e coesão nas políticas urbanas no Rio de Janeiro: Revan/ Fapesp, 2000.

Redes sociais, instituições e atores políticos no governo da cidade de São Paulo. AnnaBlume/Fapesp, 2003.

; TORRES, H. (Orgs.) São Paulo: segregação, pobreza e desigualdades sociais. São Paulo: Senac, 2005.

; $\quad$; SARAIVA, C. Favelas no município de São PauTo: estimando a sua presença para os anos de 1991, 1996 e 2000. Revista Brasileira de Estudos Urbanos, São Paulo, v. 5, n. 2, 2003.

MENEZES-FILHO, N.; PAZELLO, E. Do teachers' wages matter for proficiency? Evidences from a funding reform in Brazil. [S.1.], 2005. Mimeo.

MOSER, C. The asset vulnerability framework: reassessing urban poverty reduction strategies. World Development, [S.l.], v. 26, n.1, p 1-19, 1998.

PUTNAM, R.D. Bowling alone. New York: Touchstone, 2000

SADER, E. Quando novos personagens entram em cena. São Paulo: Paz e Terra, 1988.

SANTOS, C. Loteamentos na periferia metropolitana. Revista de Administração Municipal, Rio de Janeiro, v. 32, n. $174,1985$.

TORRES, H. et al. A pesquisa sobre segregação: conceitos, métodos e medições. Espaço \& Debates, São Paulo, v. 24 n. 45 , p. 87-109, jan./jul., 2004.

VETTER, D.; MASSENA, R. Quem se apropria dos benefícios líquidos dos investimentos do Estado em infraestrutura? Uma teoria da causação circular. In: SILVA, L. A. Machado da (Org). Solo urbano - tópicos sobre o uso da terra. [S.l., s.n.], 1981. (Série: debates urbanos).

VILLACA, F. Espaço intra-urbano no Brasil. São Paulo: Ed. Nobel, 2000.

WATSON, G. Water and sanitation in São Paulo, Brazil: successful strategies for service provision in low-income communities. 1992. Cambridge: Dissertação (Mestrado) Massachussets Institute of Technology.

WORLD BANK Entering the 21st century: world development report 1999/2000. Oxford University Press, 1999.

YINGER, J. Housing discrimination and residential segregation as causes of poverty. In: DANZIGER, S.; HAVEMAN, R. (Eds.). Understanding poverty. New York: Russell Sage Foundation; Cambridge: Harvard University Press, 2001 


\section{ANEXO I - SURVEY}

Com o objetivo de investigar diversos aspectos das condições de vida da população mais pobre do município de São Paulo, destacando especialmente as condições de acesso a políticas públicas, além de aspectos relacionados à inserção dessa população no mercado de trabalho, bem como seu comportamento político e sua participação na vida associativa, o Centro de Estudos da Metrópole (CEM-CEBRAP/CEPID-FAPESP) realizou, em parceria com o IBOPE, um survey entre os 40\% mais pobres do município. O questionário aplicado incluiu questões detalhadas para cobrir cada um desses aspectos. Essas informações contribuem para entender como as políticas públicas chegam à ponta, ou seja, nas camadas menos favorecidas da população, e não poderiam ser obtidas de maneira tão detalhada por meio de outras metodologias.

O survey realizado é representativo da população de baixa renda residente em diferentes tipos de áreas do município de São Paulo. Como população de baixa renda, consideramos os $40 \%$ mais pobres da população residente no município de São Paulo, o que correspondeu, em valores de novembro de 2004, à população com renda familiar de no máximo R\$1.100. ${ }^{9}$ Esse corte nos 40\%

${ }^{9}$ Esse corte relativo aos $40 \%$ mais pobres foi realizado com base nos dados da Pesquisa Nacional por Amostra de Domicílios (PNAD) 2002, e os valores foram atualizados para novembro de 2004 , data de realização do survey.
$2000 .^{10}$ Os três tipos de área considerados foram: áreas predominantemente habitadas por pobres, de agora em diante denominadas "macro-região periférica”, áreas de classe média (“macro-região intermediária”) e áreas habitadas predominantemente pela classe alta ("macro-região central"). ${ }^{11}$ Essas denominações estão relacionadas à leitura da distribuição geográfica dessas áreas, conforme apresentado no Mapa 1. Na Tabela 1, apresentamos a renda familiar per capita média de cada tipo de macro-região.

Tabela 1 - Renda domiciliar per capita segundo macro-regióes. Município de São Paulo, 2000

\begin{tabular}{l|cc|cc}
\hline \multirow{2}{*}{ Macro-regiões } & \multicolumn{2}{|c|}{$\begin{array}{r}\text { Renda domiciliar } \\
\text { per capita }(\mathrm{R} \$)\end{array}$} & \multicolumn{2}{|c}{$\begin{array}{r}\text { Renda domiciliar per } \\
\text { capita }(\mathrm{SM})\end{array}$} \\
\cline { 2 - 5 } & Média & $\begin{array}{c}\text { Desvio } \\
\text { padrão }\end{array}$ & Média & $\begin{array}{c}\text { Desvio } \\
\text { padrão }\end{array}$ \\
\hline Central & 11,26 & 4,93 & $1.700,15$ & 743,64 \\
\hline Intermediária & 3,98 & 1,83 & 601,37 & 276,88 \\
\hline Periférica & 2,13 & 0,94 & 321,72 & 142,38 \\
\hline Total & 4,62 & 4,29 & 697,29 & 648,33
\end{tabular}

Fonte: Microdados da Amostra do Censo Demográfico 2000, IBGE. Nota: Valores de julho de 2000.

Em cada uma dessas áreas, foi selecionada uma amostra em dois estágios. No primeiro estágio, foram sorteadas áreas de ponderação com probabilidade proporcional ao número de domicílios; no segundo, foram selecionados domicílios dentro de cada uma das áreas de ponderação sorteadas. O tamanho da amostra foi definido em 1.500 entrevistas, com 500 domicílios sorteados em cada um dos três tipos de macro-região, sendo realizada uma amostragem por cotas, considerando-se posição no domicílio (chefe ou cônjuge do

${ }^{10}$ As áreas de ponderação são unidades geográficas formadas por agrupamentos mutuamente exclusivos de setores censitários. Essas unidades são utilizadas para a aplicação dos questionários da Amostra do Censo do Censo Demográfico do IBGE, que são aplicados a $10 \%$ da população.

${ }^{11}$ Essas áreas foram delimitadas a partir de uma análise de clusters, que teve como principais variáveis a renda domiciliar média e os padrões de votação observados em cada uma dessas áreas. Para maiores detalhes dessa análise fatorial, ver Marques e Torres, 2005. Pesquisa sobre distribuição de votos na cidade mostrou também um padrão differenciado para cada uma dessas regiões. Para maiores informações, ver Figueiredo et al, 2002. 
sexo masculino - 50\% - e chefe ou cônjuge do sexo feminino, com $50 \%$ dos questionários), idade (30\% dos questionários aplicados a pessoas de 18 a 29 anos; $45 \%$ a pessoas entre 30 e 49 anos e $25 \%$ a pessoas com 50 anos ou mais) e renda familiar per capita (com $40 \%$ dos questionários aplicados a pessoas com renda entre 0 e 519 reais e $60 \%$ a pessoas com renda entre 520 e 1.100 reais). ${ }^{12}$

Somente chefes de domicílio ou cônjuges foram entrevistados, e eles forneceram informações sobre outros membros da família, quando necessário. Assim chefes ou cônjuges do município de São Paulo constituem o chamado universo da pesquisa. Além disso, foi estabelecido que seriam

Mapa 1 - Áreas de ponderação da Amostra do Censo classificadas segundo macro-regiões. Município de São Paulo, 2000

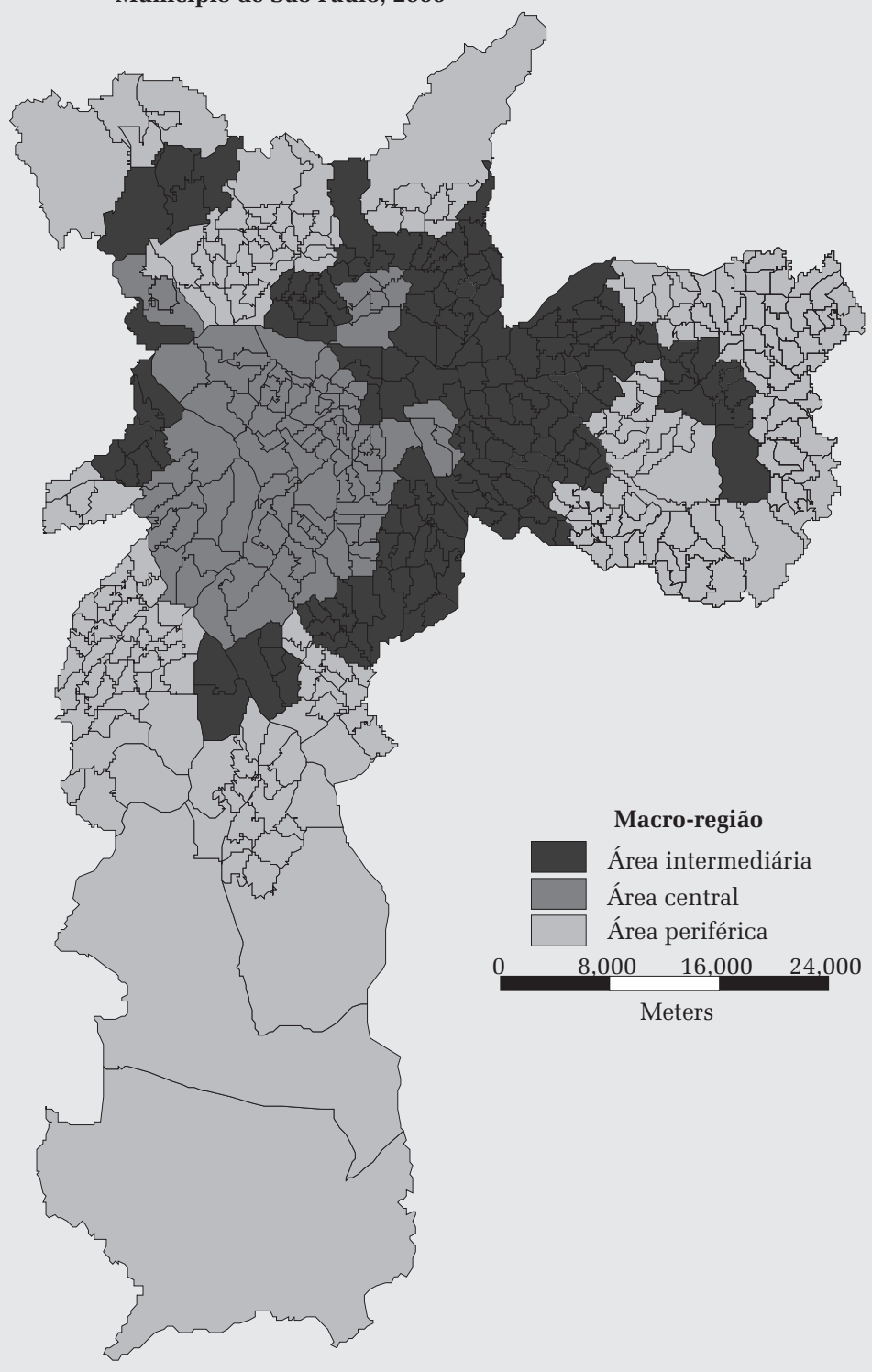

Fonte: Microdados da Amostra do Censo Demográfico 2000, IBGE.

${ }^{12}$ Essas cotas foram definidas a partir de uma análise de dados da PNAD 2002. 
realizados, no máximo, 25 questionários por área de ponderação.

Todos os questionários aplicados seguindo esses procedimentos foram posteriormente geocodificados com o recurso ao software Maptitude 4.5. ${ }^{13} \mathrm{O}$ endereçamento foi realizado por meio do CEP a 8 dígitos, e não pelo endereço completo, o que permitiu manter o sigilo dos entrevistados e, ao mesmo tempo, cruzar as informações oriundas do survey com informações oriundas de outras fontes, como os dados censitários relativos ao local de residência e a base de favelas desenvolvida pelo CEM (Marques, Torres e Saraiva, 2003), entre outros.

Para a expansão da amostra, foram criados pós-estratos, baseados nas variáveis utilizadas na criação das cotas, e para as quais se conheciam os totais populacionais. Assim, utilizando-se os dados do Censo 2000 (IBGE), foram obtidos os totais populacionais para o universo pesquisado. Dentro de cada uma das macro-regiões consideradas, a população de chefes e cônjuges (universo da pesquisa) foi dividida em 12 grupos de sexo, idade e renda familiar mensal. O produto final da aplicação desta metodologia é um fator de expansão para cada um dos questionários da amostra, cujo valor é determinado por:

${ }^{13}$ Somente 47 questionários não puderam ser endereçados, por problemas na declaração do CEP. ésimo estrato

$k=1,2, \ldots, 12$

$P_{j k}=$ peso atribuído ao $k$-ésimo grupo do $j$ -

$N_{i j k}=$ número total de pessoas do $k$-ésimo grupo do $j$-ésimo estrato

$n_{i j k}=$ número total de entrevistas do $k$-ésimo

grupo do j-ésimo estrato
Assim, após a expansão da amostra, ficamos com um universo de 1.818.422 indivíduos, correspondente aos chefes de domicílio ou cônjuges de baixa renda (no máximo $\mathrm{R} \$ 784,00$ reais em valores de julho de 2000), acima de 18 anos.

Entretanto, observa-se que, para a análise de políticas específicas, esse total varia de acordo com o recorte analítico: por exemplo, na parte relativa às políticas de educação, o conjunto de informantes refere-se aos chefes ou cônjuges de domicílios pobres que possuem jovens cursando o ensino fundamental; na parte de saúde, esse conjunto refere-se aos chefes ou cônjuges de domicílios pobres, no qual algum membro da família teve de recorrer a atendimento básico de saúde no último ano.

Também é importante ressaltar que os questionários foram aplicados aos chefes ou cônjuges e não se referem a cada um dos membros do domicílio; desse modo, os próprios chefes ou cônjuges responderam questões relativas às condições da escola de seus filhos - no caso da seção relativa ao acesso ao ensino fundamental - e também sobre o atendimento de saúde obtido no último ano por qualquer um dos membros de sua casa, no caso do acesso a serviços públicos de saúde. Apesar de essa estratégia poder apresentar limitações, acreditamos que os resultados são bastante satisfatórios, considerando os objetivos do projeto. A estratégia alternativa, ou seja, o emprego de questões referentes a cada um dos membros da família, aumentaria substancialmente os custos da pesquisa. 


\section{ANEXO II - ÁRVORE DE CHAID}

A técnica CHAID (Chi-Square Automatic Detector) permite classificar hierarquicamente os indivíduos por meio de um modelo log-linear. A partir de uma tabela de dupla entrada entre a variável preditora e a dependente, o modelo testa todas as partições possíveis das categorias da variável preditora, procurando aquela que apresenta o maior valor para a estatística qui-quadrado. A partir da escolha da partição, os dados são agrupados segundo essa partição, e uma nova análise é realizada dentro de cada subgrupo repetindo-se o procedimento anterior para a variável dependente e os demais preditores.

Estatisticamente, cada agrupamento é gerado a partir de um teste de independência entre a variável resposta (Y) e a variável preditora (X), em uma tabela de dupla entrada. No caso de Y ser nominal a hipótese alternativa é dada por:

$$
\mathrm{H}_{\mathrm{a}}: \ln \left(\frac{\mathrm{E}_{\mathrm{ij}}}{\mathrm{Z}_{\mathrm{ij}}}\right)=\lambda+\lambda_{\mathrm{i}}^{\mathrm{X}}+\lambda_{\mathrm{j}}^{\mathrm{Y}}+\lambda_{\mathrm{ij}}^{\mathrm{XY}}, \text { com Eij }
$$

$\mathrm{H}_{\mathrm{a}}: \ln \left(\frac{\mathrm{E}_{\mathrm{ij}}}{\mathrm{Z}_{\mathrm{ij}}}\right)=\lambda+\lambda_{\mathrm{i}}^{\mathrm{X}}+\lambda_{\mathrm{j}}^{\mathrm{Y}}+\mathrm{a}_{\mathrm{i}}\left(\mathrm{b}_{\mathrm{i}}-\mathrm{b}\right){ }_{\text {correspondendo ao valor esperado para a casela } \mathrm{ij}}$ sob a hipótese nula e Zij = 1/Wij (Wij representa a média dos pesos amostrais). A hipótese nula é dada por: $\mathrm{H}_{0}: \lambda_{\mathrm{ij}}^{\mathrm{XY}}=0$, que representa a independência entre $\mathrm{X}$ e Y. A estatística do teste é o $\chi^{2}$. No caso de Y ser ordinal, a hipótese alternativa é escrita como:
, com bi

corresponde ao escore da primeira categoria de Y, $\overline{\mathrm{b}}$ corresponde à média dos escores e ai um parâmetro desconhecido para a categoria yj de Y. A hipótese nula é dada por:

$\mathrm{H}_{0}: \mathrm{a}_{1}=\mathrm{a}_{2}=\ldots=\mathrm{a}_{1}$, com $\mathrm{l}$ igual ao número de categorias de $\mathrm{X}$, o que é equivalente a um teste de médias. E a estatística do teste é a razão de verossimilhança.

Operacionalmente, o procedimento pode ser descrito como:

1a Etapa: Para cada variável preditora (X), é construída uma tabela de contingência de dupla entrada, com a variável resposta (Y). Para todas as combinações possíveis das categorias da variável X é calculada a estatística $\chi$ e selecionada a combinação na qual o nível descritivo do teste (p-value) é menor do que o valor crítico ( ) pré-especificado. Escolhe-se a partição com o menor p-value.

2a Etapa: Em cada segmento criado, repetese o procedimento descrito na etapa 1.

Alguns parâmetros devem ser fixados para a realização dos testes de hipóteses. No presente caso, especificou-se uma valor crítico $(\alpha)$ de 0,05 , para a realização de partições exigiu-se um número mínimo + de 100 casos e nenhuma partição gerada poderia ter menos de 50 casos. 


\section{CONSEQÜÊNCIASDA SEGREGAÇÃO RESIDENCIAL PARA AS POLITICAS PÚBLICAS: 0 caso do atendimento básico em saúde em São Paulo}

\section{Haroldo da Gama Torres Renata Bichir}

$\mathrm{O}$ artigo procura avaliar as conseqüências da segregaç̃o residencial, ou conseq grupos sociais, para as condições de aces- concentration of certain social groups,
gruce so da população mais pobre do municí- for the conditions of access of the poorer pio de São Paulo à política de saúde, population of the city of São Paulo to the focando especialmente sobre as condi- health policies, especially focusing on the ções de acesso ao atendimento básico de conditions of access to the basic health saúde. Dialogando com abordagens que service. Dialoguing with approaches that procuram explicar as condições diferen- try to explain the differentiated ciadas de acesso a políticas públicas, o conditions of access to public policies, artigo argumenta que variáveis de diver- this paper argues that several different sas naturezas - demográficas, variables - demographic, institutional, institucionais, espaciais, relativas ao spatial, relative to associativism, among associativismo, entre outras - devem ser other - should be considered, to build a consideradas, de modo a construir um more complete scenario of the situations cenário mais completo das situações que that condition the access to public policondicionam o acesso às políticas públi- cies. For such, the authors were based cas. Para tanto, os autores basearam-se on a survey accomplished by the Center em um survey realizado pelo Centro de of Studies of the Metropolis (CEMEstudos da Metrópole (CEM-CEBRAP) CEBRAP) in November of 2004, that em novembro de 2004, que serviu de served as instrument for the evaluation tica.

CONSEQUENCES OF THE RESIDENTIAL case of the basic health service in São Paulo instrumento para a avaliação dessa polí- of that policy.

\section{LESS CONSÉQUENCES DE LA} SÉGRÉGATION RESIDENTIELLE POUR ES POLITIQUES PUBLIQUES: le cas des services de base concernant la santé à São Paulo

Haroldo da Gama Torres Renata Bichir

L'article essaie d'évaluer les conséquences de la ségrégation résidentielle, c'est-à-dire de la concentration spatiale de certains groupes sociaux en ce qui concerne les conditions d'accès aux politiques de la santé de la population plus pauvre de la municipalité de Sao Paulo. A partir des approches qui cherchent à expliquer les conditions différenciées d'accès aux politiques publiques, l'argumentation présentée dans cet article montre que des variables de natures différentes - démographiques, institutionnelles, spatiales, relatives à l'associativité, entre autres - doiven être prises en considération afin de construire un scénario plus complet des situations qui conditionnent l'accès aux politiques publiques. Pour cela, les auteurs se sont basés sur un survey réalisé par le Centre d'Etudes de la Métrople - Centro de Estudos da Metrópole (CEM-CEBRAP), en novembre 2004, et qui a été utilisé pour l'évaluation de cette politique.

KEYWORDS: public policies, health polici- MOST-CLÉs: politiques publiques, politiques PALAVRAS-CHAVE: políticas públicas, políti- es, urban segmentation, poverty and concernant la santé, segmentation

cas de saúde, segmentação urbana, po- inequalities, São Paulo. $\quad$ urbaine, pauvreté et inégalités, São Paulo. breza e desigualdades, São Paulo.

Haroldo da Gama Torres - Economista, Doutor em Ciências Sociais pela Universidade de Campinas- UNICAMP, tendo sido bolsista do Harvard Center for Population and Development Studies, durante seu programa de doutorado. Mestre em Demografia pela Universidade Federal de Minas Gerais. Concentrou seus interesses acadêmicos nas áreas de planejamento urbano, sociologia urbana e política social. Nos últimos dez anos publicou vários capítulos de livros e artigos em periódicos nacionais e internacionais, além de participar da organização de dois livros.

Renata Bichir - Doutoranda no Programa de Pós Graduação em Ciência Política do IUPERJ, Mestre em Ciência Política pelo Departamento de Ciência Política da USP e pesquisadora do Centro de Estudos da Metrópole do CEBRAP. Autora de artigos nas áreas de políticas públicas e sociologia urbana, entre eles FIGUEIREDO, Argelina; TORRES, Haroldo; BICHIR, Renata (2006). "A conjuntura social brasileira revistada". In: Novos Estudos CEBRAP, (75), julho de 2006, p. 173-184. 